\title{
Dust and Gas in the Small Magellanic Cloud
}

\author{
Tj.R. Bontekoe ${ }^{1}$ \\ Bontekoe Data Consultancy, Herengracht 2-B, 2312 LD Leiden, The \\ Netherlands \\ D.J.M. Kester \\ Space Research Organisation Netherlands, P.O. Box 800, $9700 \mathrm{AV}$ \\ Groningen, The Netherlands
}

S. Stanimirović ${ }^{2}$

University of Western Sidney Nepean, P.O. Box 10, Kingswood, NSW 2747, Australia

L. Staveley-Smith

Australia Telescope National Facility, CSIRO, P.O. Box 76, Epping, NSW 2121, Australia

J.M. van der Hulst

Kapteyn Astronomical Institute, P.O. Box 800, 9700 AV Groningen, The Netherlands

\section{High resolution IRAS images of the SMC}

Here we present four IRAS high resolution images of the Small Magellanic Cloud, as reconstructed by the HIRAS program using the Pyramid Maximum Entropy method (Bontekoe et al. 1994). (HIRAS is not to be confused with HiRes (Aumann et al. 1990).) The images show much more detail than in Schwering \& Israel (1990). Dust temperatures range from $25-41 \mathrm{~K}$ (Stanimirović et al. in prep).

\section{References}

Aumann, H.H., Fowler, J.W., \& Melnyk, M. 1990, AJ, 99, 1674

Bontekoe Tj.R., Koper E., \& Kester D.J.M. 1994, A\&A, 284, 1037

Schwering, P.B.W. \& Israel, F.P. 1990, Atlas and catalogue of infrared sources in the Magellanic clouds, Kluwer Academic Publishers.

\footnotetext{
${ }^{1}$ Astronomical Institute "Anton Pannekoek", Unversity of Amsterdam, Kruislaan 403, 1098 SJ Amsterdam, The Netherlands

${ }^{2}$ Australia Telescope National Facility, CSIRO, P.O. Box 76, Epping, NSW 2121, Australia
} 


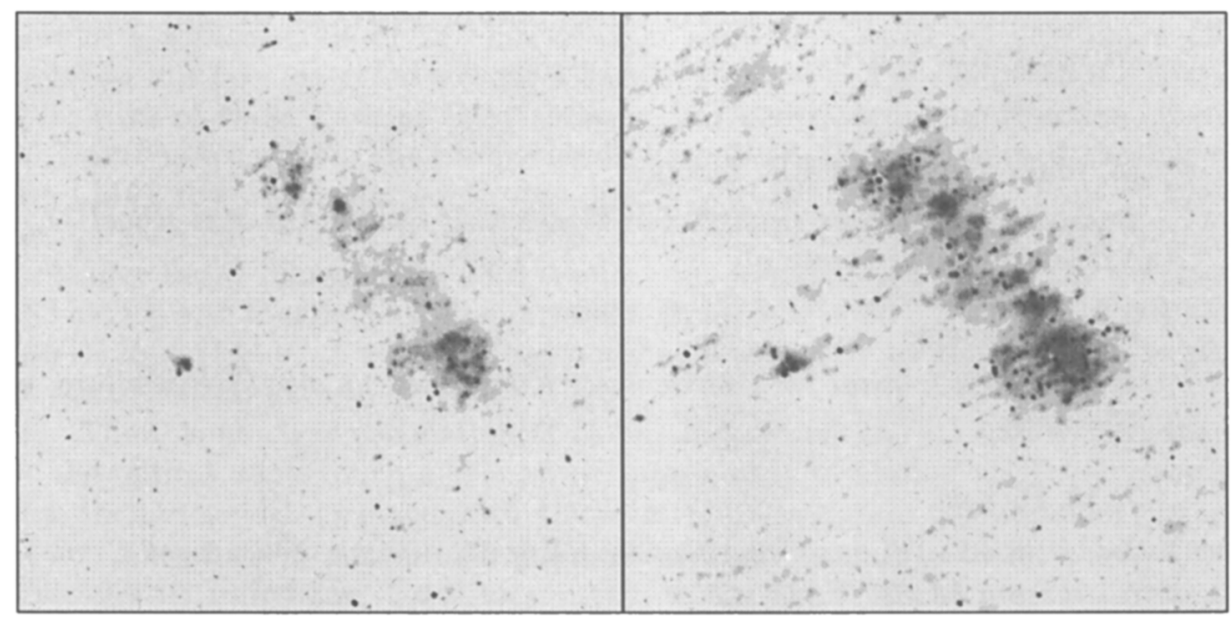

Figure 1. IRAS high resolution images of SMC, $12 \mu \mathrm{m}$ (left) and $25 \mu \mathrm{m}$. Spatial resolution of both images is about $1^{\prime}$. Grey scale is identical for both images (0.3 0.4 0.5 0.6 0.8 $125101520 \mathrm{MJy} / \mathrm{Sr})$.

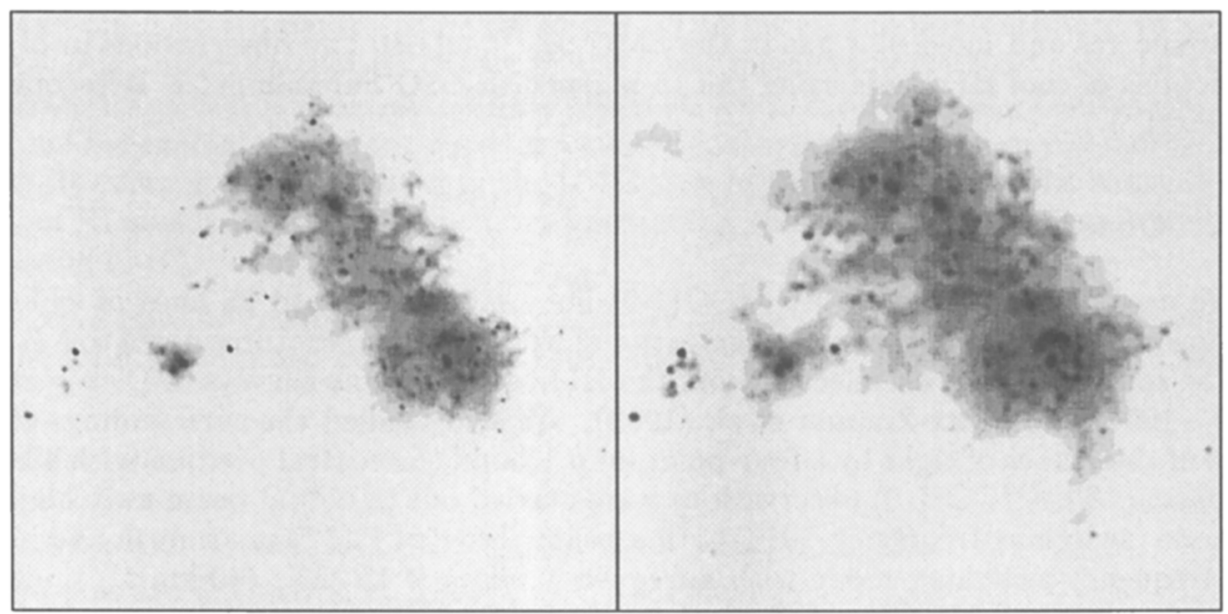

Figure 2. IRAS high resolution images of SMC, $60 \mu \mathrm{m}$ (left) and $100 \mu \mathrm{m}$. Spatial resolutions are about $1^{\prime}$ and $1.7^{\prime}$ resp. Grey scale

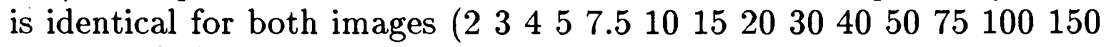
$200 \mathrm{MJy} / \mathrm{Sr}$ ). 